\title{
THE CHARACTERIZATION OF FUNCTIONS ARISING AS POTENTIALS. II
}

\author{
BY E. M. STEIN ${ }^{1}$
}

Communicated by A. Zygmund, August 8, 1962

1. Statement of result. We continue our study of the function spaces $L_{\alpha}^{p}$, begun in [7]. We recall that $f \in L_{\alpha}^{p}\left(E_{n}\right)$ when $f=K_{\alpha} * \phi$, where $\phi \in L^{p}\left(E_{n}\right) . K_{\alpha}$ is the Bessel kernel, characterized by its Fourier transform $K_{\alpha}(x)^{\wedge}=\left(1+|x|^{2}\right)^{-\alpha / 2}$. It should also be recalled that the space $L_{k}^{p}, 1<p<\infty$, with $k$ a positive integer, coincides with the space of functions which together with their derivatives up to and including order $k$ belong to $L^{p}$; (see [2]).

It will be convenient to give the functions in $L_{\alpha}^{p}$ their strict definition. Thus we redefine them to have the value $\left(K_{\alpha} * \phi\right)(x)$ at every point where this convolution converges absolutely. With this done, and if $\alpha-(n-m) / p>0$, then the restriction of an $f \in L_{\alpha}^{p}\left(E_{n}\right)$ to a fixed $m$-dimensional linear variety in $E_{n}$ is well-defined (that is, it exists almost everywhere with respect to $m$-dimensional Euclidean measure). The problem that arises is of characterizing such restrictions.

The problem was previously solved in the following cases:

(i) When $p$ is arbitrary, but $\alpha=1$, in Gagliardo [3].

(ii) When $p=2$, and $\alpha$ is otherwise arbitrary in Aronszajn and Smith [1]. In each case the solution may be expressed in terms of another function space, $W_{\alpha}^{p}$, which consists of those $f \in L^{p}\left(E_{n}\right)$ for which the norm ${ }^{2}$

$$
\|f\|_{p}+\left[\int_{E_{n}} \int_{E_{n}} \frac{|f(x-y)-f(x)|^{p}}{|y|^{n+\alpha p}} d x d y\right]^{1 / p}
$$

is finite, when $0<\alpha<1$. When $0<\alpha<2$, there is a similar definition of $W_{\alpha}^{p}$ (consistent with the previous one for $0<\alpha<1$ ) which replaces the difference $f(x-y)-f(x)$ by the second difference $f(x-y)+f(x+y)$ $-2 f(x)$. Finally for general $\alpha \geqq 2$, the spaces $W_{\alpha}^{p}$ are defined recurrently by $f \in W_{\alpha}^{p}$ when $f \in L^{p}$ and $\partial f / \partial x_{n} \in W_{\alpha-1}^{p}, k=1, \cdots, n$.

In stating our result we let $E_{m}$ denote a fixed proper $m$ dimensional subspace of $E_{n}$, and $R f$ denote the restriction to $E_{m}$ of a function defined on $E_{n}$.

\footnotetext{
1 The author wishes to acknowledge the support of the Alfred P. Sloan Foundation.

${ }^{2}$ Such norms were considered when $n=1$ in [5]. The space is also considered in [6] and [9]; in the latter it is denoted by $\Lambda_{\alpha}^{p . p}$.
} 
THEOREM. (a) The restriction mapping $R$ is continuous from $L_{\alpha}^{p}\left(E_{n}\right)$ to $W_{\beta}^{p}\left(E_{m}\right)$, if $\beta=\alpha-(m-n) / p$, as long as $\beta>0$, and $1<p<\infty$.

(b) Conversely, there exists a linear extension mapping $\mathcal{E}$, defined on functions of $E_{m}$ to function of $E_{n}$, so that $\varepsilon$ is continuous from $W_{\beta}^{p}\left(E_{m}\right)$ to $L_{\alpha}^{p}\left(E_{n}\right)$, and $R(\mathcal{E}(g))=g$ for every $g \in W_{\beta}^{p}\left(E_{m}\right)$, as long as $\beta>0$ and $1<p<\infty .^{3}$

It should be pointed out that the spaces $L_{\alpha}^{p}$, when either $\alpha$ is integral or $p=2$, are in some sense exceptional. Only in these cases can the elements of $L_{\alpha}^{p}$ be characterized in terms of the $L^{p}$ modulus of continuity (i.e. conditions bearing on $\|f(x-y)-f(x)\|_{p}$ when say $0<\alpha \leqq 1$ ). In particular, $L_{\alpha}^{p}$ is equivalent with $W_{\alpha}^{p}$ only if $p=2$; see Taibleson [9]. It is known ${ }^{4}$ that the restrictions of $W_{\alpha}^{p}\left(E_{n}\right)$ are elements of $W_{\beta}^{p}\left(E_{m}\right)$ with $\beta=\alpha-(n-m) / p$. As we shall see, this result is an immediate consequence of our theorem. Thus we have the interesting situation of two different spaces, $L_{\alpha}^{p}$ and $W_{\alpha}^{p}$, having identical restriction spaces.

2. Proof of the Theorem. What follows is a sketch of the proof, details omitted. We consider the case $m=n-1,0<\alpha<1$; the general case is dealt with similarly. We shall make consistent use of the following notation: latin letters, $x, y, z, \cdots$ will stand for variables of $E_{n-1}$ considered as a subspace of $E_{n}$; greek letters $\xi, \eta, \zeta, \cdots$ for points in $E_{1}$, which is the orthogonal subspace. Thus the pair $(x, \xi)$ belongs to $E_{n}$. Also if $f(x, \xi)$ is a function defined on $E_{n}$, then $\|f(\cdot, \xi)\|_{p}$ will denote $L^{p}$ norm with respect to the $x$ variable, $\xi$ fixed; $\|f(\cdot, \cdot)\|_{p}$ will denote the norm taken over both variables. Using the same convention, $\|g(\cdot+y)-g(\cdot)\|_{p}$ will stand for

$$
\left(\int_{-\infty}^{+\infty}|g(x+y)-g(x)|^{p} d x\right)^{1 / p} .
$$

We make consistent use of the following classical estimate [4].

Lemma. If $\Phi(\xi)=\int_{0}^{\infty} K(\xi, \eta) \phi(\eta) d \eta$, where $K$ is homogeneous of degree -1 , then $\int_{0}^{\infty}|\Phi(\xi)|^{p} d \xi \leqq A^{p} \int_{0}^{\infty}|\phi(\eta)|^{p} d \eta$, where

$$
A=\int_{0}^{\infty}|K(1, \eta)| \eta^{-1 / p} d \eta<\infty .
$$

Now suppose that $f \in L_{\alpha}^{p}\left(E_{n}\right)$; then $f=K_{\alpha} * \phi$ where $\phi \in L^{p}\left(E_{n}\right)$; and the norm of $f$ in $L^{p},\|f\|_{p, \alpha}$, is given by $\|f\|_{p, \alpha}=\|\phi\|_{p}$. Let $g=R(f)$. Then

3 The mapping $\mathcal{E}$ is defined on all locally integrable functions of $E_{m}$.

4 This result is due to several Soviet authors. For references see [9], and the paper of O. V. Besov in Trudy Steklov Inst. Acad. Sci. USSR 60 (1961), 42-81. 


$$
g(x)=\int_{-\infty}^{\infty} \int_{E_{n-1}} \phi(x-z, \xi) K_{\alpha}(z, \xi) d z d \xi
$$

Hence,

$$
|g(x)| \leqq \int_{E_{n-1}}\|\phi(x-z, \cdot)\|_{p}\|K(z, \cdot)\|_{q} d z
$$

where $1 / p+1 / q=1$. From this it follows that

$$
\|g\|_{p} \leqq\|\phi(\cdot, \cdot)\|_{p} \int_{E_{n-1}}\|K(z, \cdot)\|_{\alpha} d z=A\|\phi\|_{p}=A\|f\|_{p, \alpha} .
$$

This is a consequence of the fact that $\int_{E_{n-1}}\left\|K_{\alpha}(z, \cdot)\right\|_{q} d z<\infty$ if $\alpha-1 / p>0$, which follows easily from the estimates

$$
K_{\alpha}(z, \xi)=O\left(|z|^{2}+\xi^{2}\right)^{(-n+\alpha) / 2} \quad \text { for }|z|^{2}+\xi^{2} \rightarrow 0,
$$

and

$$
K_{\alpha}(z, \xi)=O\left(\exp -\frac{\left(|z|^{2}+\xi^{2}\right)^{1 / 2}}{2}\right) \quad \text { for }|z|^{2}+\xi^{2} \rightarrow \infty ;
$$

see [1].

Next, define $g_{\xi}(x)$ by $g_{\xi}(x)=\int_{E_{n-1}} \phi(x-z, \xi) K_{\alpha}(z, \xi) d z$. Thus $g(x)$ $=\int g_{\xi}(x) d \xi$. We have

$$
\left\|g_{\xi}(\cdot+y)-g_{\xi}(\cdot)\right\|_{p} \leqq\|\phi(\cdot, \xi)\|_{p} \int_{E_{n-1}}\left|K_{\alpha}(z-y, \xi)-K_{\alpha}(z, \xi)\right| d z .
$$

Using the fact, (see, [1]) that $\nabla K_{\alpha}=O\left(|x|^{2}+\xi^{2}\right)^{(-n-1+\alpha) / 2}$ and the previous estimates on $K_{\alpha}$, it can be shown that the last integral is dominated by $A|y|^{-1+\alpha} \psi(\xi /|y|)$, where $\psi(u)=O\left(|u|^{-1+\alpha}\right)$ as $u \rightarrow 0$ and $O\left(|u|^{-2+\alpha}\right)$ as $u \rightarrow \infty,(0<\alpha<1$, here). From this it follows that $\|g(\cdot-y)-g(\cdot)\|_{p}$

$$
\leqq A\left[\int_{|\xi| \leq|y|}|\xi|^{-1+\alpha}\|\phi(\cdot, \xi)\|_{p} d \xi+|y| \int_{|\xi| z|y|}|\xi|^{-2+\alpha \|}\|\phi(\cdot, \xi)\|_{p} d \xi\right] .
$$

An application of the lemma then shows, since $1>\alpha>1 / p$,

$$
\begin{aligned}
\int \frac{\|g(\cdot-y)-g(\cdot)\|_{p}^{p}}{|y|^{n-2+\alpha p}} d y & \leqq A \int_{-\infty}^{\infty}\|\phi(\cdot, \xi)\|_{p}^{p} d \xi \\
& =A\|\phi\|_{p}^{p}=A\|f\|_{p, \alpha}^{p} .
\end{aligned}
$$

Combining this with (1) above proves part (a) of the theorem. To 
prove the converse, assume that $g \in W_{\beta}^{p}\left(E_{n-1}\right)$, and $g$ is sufficiently smooth. The smoothness is no restriction of generality since our estimates will be seen to be uniform in the norm. To define the extension operator, choose $\psi \in C_{0}^{\infty}\left(E_{n-1}\right), \int_{E_{n-1}} \psi(x) d x=1$, and $\psi$ vanishes outside the unit sphere. Also choose $\lambda \in C_{0}^{\infty}\left(E_{1}\right)$ so that $\lambda(0)=1$.

Let

$$
\mathcal{E}(g)=f(x, \xi)=\lambda(\xi)|\xi|^{-n+1} \int_{E_{n-1}} g(x-y) \psi(y /|\xi|) d y .
$$

Notice that $f(x, 0)=g(x)$ and $\|f(\cdot, \cdot)\|_{p} \leqq A\|g\|_{p}$.

In order to prove that $f \in L_{\alpha}^{p}\left(E_{n}\right)$, we shall consider $F=J_{1-\alpha}(f)$, and show that $F \in L_{1}^{p}\left(E_{n}\right)$. This will suffice because $J_{1-\alpha}$ is a normpreserving isomorphism of $L_{\alpha}^{p}$ onto $L_{1}^{p}$. To prove $F(x, \xi) \in L_{1}^{p}\left(E_{n}\right)$ it suffices to show that $F, \partial F / \partial x_{k}, \partial F / \partial \xi$ all belong to $L^{p}\left(E_{n}\right)$. However, this is clear for $F$ itself, because $f \in L^{p}\left(E_{n}\right)$ and $J_{1-\alpha}$ does not increase the $L^{p}$ norm. Thus we consider $\partial F / \partial x_{k}$. Now

$$
\left.F(x, \xi)=\iint K_{1-\alpha}(z, \eta) f(x-z), \xi-\eta\right) d z d \eta
$$

However

$$
\left|\frac{\partial f}{\partial x_{k}}(x, \xi)\right| \leqq A|\xi|^{-n} \int_{|y| \leqq|\xi|}|g(x-y)-g(x)| d y,
$$

by (3), because

$$
\int \frac{\partial}{\partial x_{k}} \psi(x) d x=0
$$

and $\psi$ vanishes outside the unit sphere. Also, as we have seen $\left|K_{1-\alpha}(z, \xi)\right| \leqq A\left(|z|^{2}+\xi^{2}\right)^{(-n+1-\alpha) / 2}$. Therefore we see

$$
\begin{aligned}
\left|\frac{\partial}{\partial x_{k}} F(x, \xi)\right| \leqq A \int & \left(|z|^{2}+\eta^{2}\right)^{(-n+1-\alpha) / 2}|\xi-\eta|^{-n} \\
& \cdot \int_{|y| \leqq|\xi-\eta|}|g(x-y-z)-g(x-z)| d y d z d \eta
\end{aligned}
$$

Let us now set $\left.\omega(y)=\|g(\cdot-y)-g(\cdot)\|_{p},\right)$ and

$$
\Omega(\rho)=\rho^{-n+1-\alpha} \int_{|y|<\rho} \omega(y) d y, \quad 0<\rho<\infty .
$$

Then by (4) and Minkowski's inequality for integrals we get 


$$
\begin{aligned}
& \left\|\frac{\partial F}{\partial x_{k}}(\cdot, \xi)\right\|_{p} \\
& \quad \leqq A \int_{E_{n-1}} \int_{-\infty}^{\infty}\left(|z|^{2}+\eta^{2}\right)^{(-n+1-\alpha) / 2}|\xi-\eta|^{-1+\alpha} \Omega(|\xi-\eta|) d \eta d z .
\end{aligned}
$$

Carrying out the integration of $z$ over $E_{n-1}$ gives

$$
\begin{aligned}
&\left\|\frac{\partial F}{\partial x_{k}}(\cdot, \xi)\right\|_{p} \leqq A \int_{-\infty}^{+\infty}|\eta|^{-\alpha}|\xi-\eta|^{-1+\alpha} \Omega(|\xi-\eta|) d \eta \\
&=A \int_{-\infty}^{\infty}|\xi-\eta|^{-\alpha}|\eta|^{-1+\alpha} \Omega(|\eta|) d \eta .
\end{aligned}
$$

A two-fold application of the lemma then shows, since $\alpha>1 / p$,

$$
\begin{aligned}
\left\|\frac{\partial F}{\partial x_{k}}\right\|_{p} & =\left\|\frac{\partial F}{\partial x_{k}}(\cdot, \cdot)\right\|_{p} \leqq A \int_{0}^{\infty}[\Omega(\rho)]^{p} d \rho \leqq A \int_{E_{n-1}} \frac{\omega^{p}(y) d y}{|y|^{n-1+\beta_{p}}} \\
& =A \int_{E_{n-1}} \int_{E_{n-1}} \frac{|g(x-y)-g(x)|^{p}}{|y|^{n-1+\beta_{p}}} d y d x .^{5}
\end{aligned}
$$

Similar estimates hold for $\partial F / \partial \xi$. This completes the proof of the theorem.

3. Further remarks. We have the following corollary of our theorem

Corollary. (a) If $f \in L_{\alpha}^{p}\left(E_{n}\right)$, then $R f \in L_{\beta}^{p}\left(E_{m}\right)$, when $\beta=\alpha$ $-(n-m) / p>0$, and $1<p \leqq 2$.

(b) If $g \in L_{\beta}^{p}\left(E_{m}\right), \quad \beta=\alpha-(n-m) / p>0$, then $\mathcal{E}(g) \in L_{\alpha}^{p}\left(E_{n}\right)$, if $2 \leqq p<\infty$.

Part (a) of the corollary is due to Calderon [2]. Part (b) is its appropriate converse. The corollary follows from the theorem and the known continuous inclusion relations $W_{\alpha}^{p} C L_{\alpha}^{p}$, if $1 \leqq p \leqq 2$, and $L_{\alpha}^{p} \subset W_{\alpha}^{p}$ if $2 \leqq p \leqq \infty$; see Taibleson [9].

We shall now point out how to obtain an analogue of our theorem which replaces $L_{\alpha}^{p}\left(E_{n}\right)$ by $W_{\alpha}^{p}\left(E_{n}\right)$. Thus let $f \in W_{\alpha}^{p}\left(E_{n}\right)$. By part (b) of the theorem it has an extension to a function in $E_{n+1}$ which belongs to $L_{\alpha+1 / p}^{p}\left(E_{n+1}\right)$. By part (a) this extension, when restricted to $E_{m}$, belongs to $W_{\beta}^{p}\left(E_{m}\right)$, where $\beta=\alpha-(n-m) / p$. However this restriction is obviously the restriction of our original $f$. Therefore the restriction of

${ }^{5}$ To prove this one may also use an $n$-dimensional variant of the lemma; see [8, Lemma (3.5)]. 
an $f \in W_{\boldsymbol{\alpha}}^{p}\left(E_{n}\right)$ belongs to $W_{\beta}^{p}\left(E_{m}\right)$. In the same way the analogous extension property is proved.

\section{REFERENCES}

1. N. Aronszajn and K. T. Smith, Theory of Bessel potentials. I, Ann. Inst. Fourier 11 (1961), 385-475. (Reference [1] in paper [7] below.

2. A. P. Calderon, Lebesgue spaces of functions and distributions, Proc. Sympos. Pure Math. Vol. 4, pp. 33-49, Amer. Math. Soc., Providence, R. I., 1961. (Reference [2] in paper [7] below.

3. E. Gagliardo, Caratterizzazioni delle trace sulla frontiera relative ad alcune classi di funzioni in $n$ variabili, Rend. Sem. Mat. Univ. Padova 27 (1957), 284-305.

4. G. H. Hardy, J. E. Littlewood and G. Polya, Inequalities, Cambridge, 1934.

5. I. I. Hirschman, Fractional integration, Amer. J. Math. 75 (1953), 531-546.

6. L. N. Slobodetzky, Dokl. Akad. Nauk SSSR 118 (1958), 243-246; 120 (1958), 468-471. (Russian)

7. E. M. Stein, The characterization of functions arising as potentials, Bull. Amer. Math. Soc. 67 (1961), 102-104.

8. E. M. Stein and G. Weiss, Fractional integrals on n-dimensional Euclidean space, J. Math. Mech. 7 (1958), 503-514.

9. M. Taibleson, Smoothness and differentiability conditions for functions and distributions in $E_{n}$, Dissertation, University of Chicago, 1962.

University of Chicago 